Supporting Information

Why the Reactive Oxygen Species of the Fenton Reaction Switches from Oxoiron(IV) Species to Hydroxyl Radical in Phosphate Buffer Solutions? A Computational Rationale

\title{
Hsing-Yin Chen
}

Department of Medicinal and Applied Chemistry, Kaohsiung Medical University, Kaohsiung 80708, Taiwan

E-mail: hychen@kmu.edu.tw

\section{Table of Contents}

- Page S2. Gas-phase electronic energy change of $\left[\mathrm{Fe}^{\mathrm{II}} \mathrm{H}_{2} \mathrm{O}\right]^{2+}+\mathrm{H}_{2} \mathrm{PO}_{4}^{-} \rightarrow$ $\left[\mathrm{Fe}^{\mathrm{II}} \mathrm{H}_{2} \mathrm{PO}_{4}\right]^{+}+\mathrm{H}_{2} \mathrm{O}$.

- Page S2. Hydration energies of $\mathrm{H}_{2} \mathrm{O}$ and $\mathrm{H}_{2} \mathrm{PO}_{4}^{-}$.

- Page S3. Free energy change of successive $\mathrm{H}_{2} \mathrm{O}$ exchange by $\mathrm{H}_{2} \mathrm{PO}_{4}^{-}$for $\left[\mathrm{Fe}^{\mathrm{II}}\left(\mathrm{H}_{2} \mathrm{O}\right)_{6}\right]^{2+}$.

- Page S4. Average bond distances and standard deviations for ADMP dynamics simulation of $\left[\mathrm{Fe}^{\mathrm{II}}\left(\mathrm{H}_{2} \mathrm{PO}_{4}\right)_{5}\left(\mathrm{H}_{2} \mathrm{O}\right)\right]^{3-}$.

- Page S5. Calculated $\left\langle S^{2}\right\rangle$ values.

- Page S6-S7. ADMP dynamics simulations of $\left[\mathrm{Fe}^{\mathrm{II}}\left(\mathrm{H}_{2} \mathrm{PO}_{4}\right)_{5}\left(\mathrm{H}_{2} \mathrm{O}\right)\right]^{3-}$. 
Table S1. Gas-phase electronic energy change (kcal/mol) of $\left[\mathrm{Fe}^{\mathrm{II}} \mathrm{H}_{2} \mathrm{O}\right]^{2+}+\mathrm{H}_{2} \mathrm{PO}_{4}^{-} \rightarrow$ $\left[\mathrm{Fe}^{\mathrm{II}} \mathrm{H}_{2} \mathrm{PO}_{4}\right]^{+}+\mathrm{H}_{2} \mathrm{O}$.

\begin{tabular}{|l|l|}
\hline method & $\Delta \mathrm{E}$ \\
\hline B3LYP-D3/6-31+G(d) & -301.7 \\
\hline PBE0-D3/6-31+G(d) & -301.9 \\
\hline M06-D3/6-31+G(d) & -303.5 \\
\hline PW6B95-D3/6-31+G(d) & -296.9 \\
\hline PW6B95-D3/Def2-TZVP & -300.3 \\
\hline PW6B95-D3/Def2-QZVP & -300.0 \\
\hline BD(T)/Def2-TZVPP & -293.4 \\
\hline
\end{tabular}

Table S2. Hydration energies ( $\mathrm{kcal} / \mathrm{mol}$ ) of $\mathrm{H}_{2} \mathrm{O}$ and $\mathrm{H}_{2} \mathrm{PO}_{4}^{-}$. Signed errors are given in parentheses. $^{a}$

\begin{tabular}{|l|l|l|}
\hline method & $\mathrm{H}_{2} \mathrm{O}$ & $\mathrm{H}_{2} \mathrm{PO}_{4}^{-}$ \\
\hline SMD/PW6B95-D3/6-31+G(d) & $-9.3(-3.0)$ & $-76.9(-8.9)$ \\
\hline IEF-PCM/PW6B95-D3/6-31+G(d) $^{d}$ & $-5.4(0.9)$ & $-67.1(0.9)$ \\
\hline CPCM/PW6B95-D3/6-31+G(d) ${ }^{d}$ & $-5.5(0.8)$ & $-67.2(0.8)$ \\
\hline Expt. & -6.3 & -68 \\
\hline
\end{tabular}

${ }^{a}$ Theoretical hydration energies were evaluated by the difference between solutionphase electronic energy and gas-phase electronic energy at solution-phase optimized geometry. 
Table S3. Free energy change $(\mathrm{kcal} / \mathrm{mol})$ of successive $\mathrm{H}_{2} \mathrm{O}$ exchange by $\mathrm{H}_{2} \mathrm{PO}_{4}{ }^{-}$for $\left[\mathrm{Fe}^{\mathrm{II}}\left(\mathrm{H}_{2} \mathrm{O}\right)_{6}\right]^{2+}$. ${ }^{a}$

\begin{tabular}{|l|l|l|l|l|l|l|}
\hline $\mathrm{n}$ & $\mathrm{CPCM} / \mathrm{PBE} 0 / 6-31+\mathrm{G}(\mathrm{d})$ & $\mathrm{SMD} / \mathrm{PBE} 0 / 6-31+\mathrm{G}(\mathrm{d})$ & $\mathrm{CPCM} / \mathrm{M} 06 / 6-31+\mathrm{G}(\mathrm{d})$ & $\mathrm{CPCM} / \mathrm{PW} 6 \mathrm{~B} 95 / 6-31+\mathrm{G}(\mathrm{d})$ & $\mathrm{CPCM} /$ PBE0/Def2-TZVP $^{b}$ & $\mathrm{CPCM} /$ PBE0/Def2-QZVP $^{b}$ \\
\hline 1 & -16.5 & -13.7 & -15.5 & -17.4 & -18.1 & -16.9 \\
\hline 2 & -14.8 & -12.9 & -14.6 & -15.2 & -16.3 & -15.4 \\
\hline 3 & -13.1 & -13.4 & -11.5 & -10.8 & -16.4 & -14.2 \\
\hline 4 & -11.8 & -13.3 & -11.0 & -13.6 & -9.4 & -7.5 \\
\hline 5 & -8.4 & -14.7 & -7.8 & -7.4 & -9.2 & -8.8 \\
\hline 6 & -6.0 & -8.0 & -5.4 & -4.1 & -5.9 & -5.3 \\
\hline
\end{tabular}

${ }^{a}$ Grimme's D3 dispersion correction was included and experimental hydration energies of $\mathrm{H}_{2} \mathrm{O}$ and $\mathrm{H}_{2} \mathrm{PO}_{4}^{-}$were employed in all calculations. ${ }^{b} \mathrm{CPCM} / \mathrm{PBE} 0 / 6-31+\mathrm{G}(\mathrm{d})$ optimized geometries are used. 
Table S4. Average bond distances and standard deviations $(\AA)$

\begin{tabular}{ccc}
\hline bond label $^{a}$ & bond distance & standard deviation \\
\hline $\mathrm{FeL}_{1}$ & 2.339 & 0.144 \\
$\mathrm{FeL}_{2}$ & 2.228 & 0.109 \\
$\mathrm{FeL}_{3}$ & 2.131 & 0.097 \\
$\mathrm{FeL}_{4}$ & 2.212 & 0.130 \\
$\mathrm{FeL}_{5}$ & 2.031 & 0.069 \\
$\mathrm{FeL}_{6}$ & 2.071 & 0.087 \\
$\mathrm{HB}_{1}$ & 2.166 & 0.518 \\
$\mathrm{HB}_{2}$ & 1.883 & 0.168 \\
$\mathrm{HB}_{3}$ & 3.101 & 0.396 \\
$\mathrm{HB}_{4}$ & 1.783 & 0.184 \\
$\mathrm{HB}_{5}$ & 1.830 & 0.199 \\
$\mathrm{HB}_{6}$ & 1.799 & 0.142 \\
$\mathrm{HB}_{7}$ & 1.701 & 0.085 \\
$\mathrm{HB}_{8}$ & 1.735 & 0.145 \\
$\mathrm{HB}_{9}$ & 1.982 & 0.183 \\
\hline
\end{tabular}

${ }^{a}$ See Figure 1 for bond label 
Table S5. Calculated $\left\langle S^{2}\right\rangle$ values.

\begin{tabular}{|c|c|c|c|}
\hline & spin state & calculated $\left\langle S^{2}\right\rangle$ & $\operatorname{exact}\left\langle S^{2}\right\rangle$ \\
\hline$\left[\mathrm{Fe}^{\mathrm{II}}\left(\mathrm{H}_{2} \mathrm{O}\right)_{6}\right]^{2+}$ & quintet & 6.0046 & 6.0000 \\
\hline$\left[\mathrm{Fe}^{\mathrm{II}}\left(\mathrm{H}_{2} \mathrm{PO}_{4}\right)\left(\mathrm{H}_{2} \mathrm{O}\right)_{5}\right]^{+}$ & quintet & 6.0048 & 6.0000 \\
\hline$\left[\mathrm{Fe}^{\mathrm{II}}\left(\mathrm{H}_{2} \mathrm{PO}_{4}\right)_{2}\left(\mathrm{H}_{2} \mathrm{O}\right)_{4}\right]$ & quintet & 6.0051 & 6.0000 \\
\hline$\left[\mathrm{Fe}^{\mathrm{II}}\left(\mathrm{H}_{2} \mathrm{PO}_{4}\right)_{3}\left(\mathrm{H}_{2} \mathrm{O}\right)_{3}\right]^{-}$ & quintet & 6.0050 & 6.0000 \\
\hline$\left[\mathrm{Fe}^{\mathrm{II}}\left(\mathrm{H}_{2} \mathrm{PO}_{4}\right)_{4}\left(\mathrm{H}_{2} \mathrm{O}\right)_{2}\right]^{2-}$ & quintet & 6.0050 & 6.0000 \\
\hline$\left[\mathrm{Fe}^{\mathrm{II}}\left(\mathrm{H}_{2} \mathrm{PO}_{4}\right)_{5}\left(\mathrm{H}_{2} \mathrm{O}\right)\right]^{3-}$ & quintet & 6.0051 & 6.0000 \\
\hline$\left[\mathrm{Fe}^{\mathrm{II}}\left(\mathrm{H}_{2} \mathrm{PO}_{4}\right)_{6}\right]^{4-}$ & quintet & 6.0051 & 6.0000 \\
\hline $\mathrm{RC}$ & quintet & 6.0059 & 6.0000 \\
\hline INT1 $_{\mathrm{OH} / \bullet \mathrm{OH}}$ & quintet & 6.9681 & 6.0000 \\
\hline INT2 $2_{\mathrm{OH} / \bullet \mathrm{OH}}$ & quintet & 7.0081 & 6.0000 \\
\hline INT $_{\text {FeIII+•OH }}$ & quintet & 7.0080 & 6.0000 \\
\hline INT $_{\text {oxo }}$ & quintet & 6.1180 & 6.0000 \\
\hline$\left[\mathrm{Fe}^{\mathrm{III}}\left(\mathrm{H}_{2} \mathrm{O}\right)_{6}\right]^{3+}$ & sextet & 8.7546 & 8.7500 \\
\hline$\left[\mathrm{Fe}^{\mathrm{III}}\left(\mathrm{H}_{2} \mathrm{PO}_{4}\right)\left(\mathrm{H}_{2} \mathrm{O}\right)_{5}\right]^{2+}$ & sextet & 8.7554 & 8.7500 \\
\hline$\left[\mathrm{Fe}^{\mathrm{III}}\left(\mathrm{H}_{2} \mathrm{PO}_{4}\right)_{2}\left(\mathrm{H}_{2} \mathrm{O}\right)_{4}\right]^{+}$ & sextet & 8.7556 & 8.7500 \\
\hline$\left[\mathrm{Fe}^{\mathrm{III}}\left(\mathrm{H}_{2} \mathrm{PO}_{4}\right)_{3}\left(\mathrm{H}_{2} \mathrm{O}\right)_{3}\right]$ & sextet & 8.7556 & 8.7500 \\
\hline$\left[\mathrm{Fe}^{\mathrm{III}}\left(\mathrm{H}_{2} \mathrm{PO}_{4}\right)_{4}\left(\mathrm{H}_{2} \mathrm{O}\right)_{2}\right]^{-}$ & sextet & 8.7560 & 8.7500 \\
\hline$\left[\mathrm{Fe}^{\mathrm{III}}\left(\mathrm{H}_{2} \mathrm{PO}_{4}\right)_{5}\left(\mathrm{H}_{2} \mathrm{O}\right)\right]^{2-}$ & sextet & 8.7559 & 8.7500 \\
\hline$\left[\mathrm{Fe}^{\mathrm{III}}\left(\mathrm{H}_{2} \mathrm{PO}_{4}\right)(\mathrm{OH})\left(\mathrm{H}_{2} \mathrm{O}\right)_{4}\right]^{+}$ & sextet & 8.7568 & 8.7500 \\
\hline$\left[\mathrm{Fe}^{\mathrm{III}}\left(\mathrm{H}_{2} \mathrm{PO}_{4}\right)_{2}(\mathrm{OH})\left(\mathrm{H}_{2} \mathrm{O}\right)_{3}\right]$ & sextet & 8.7562 & 8.7500 \\
\hline$\left[\mathrm{Fe}^{\mathrm{III}}\left(\mathrm{H}_{2} \mathrm{PO}_{4}\right)_{3}(\mathrm{OH})\left(\mathrm{H}_{2} \mathrm{O}\right)_{2}\right]^{-}$ & sextet & 8.7557 & 8.7500 \\
\hline$\left[\mathrm{Fe}^{\mathrm{III}}\left(\mathrm{H}_{2} \mathrm{PO}_{4}\right)_{4}(\mathrm{OH})\left(\mathrm{H}_{2} \mathrm{O}\right)\right]^{2-}$ & sextet & 8.7560 & 8.7500 \\
\hline$\left[\mathrm{Fe}^{\mathrm{III}}\left(\mathrm{H}_{2} \mathrm{PO}_{4}\right)_{5}(\mathrm{OH})\right]^{3-}$ & sextet & 8.7558 & 8.7500 \\
\hline$\left[\mathrm{Fe}^{\mathrm{III}}\left(\mathrm{HPO}_{4}\right)\left(\mathrm{H}_{2} \mathrm{O}\right)_{5}\right]^{+}$ & sextet & 8.7571 & 8.7500 \\
\hline$\left[\mathrm{Fe}^{\mathrm{III}}\left(\mathrm{H}_{2} \mathrm{PO}_{4}\right)\left(\mathrm{HPO}_{4}\right)\left(\mathrm{H}_{2} \mathrm{O}\right)_{4}\right]$ & sextet & 8.7561 & 8.7500 \\
\hline$\left[\mathrm{Fe}^{\mathrm{III}}\left(\mathrm{H}_{2} \mathrm{PO}_{4}\right)_{2}\left(\mathrm{HPO}_{4}\right)\left(\mathrm{H}_{2} \mathrm{O}\right)_{3}\right]^{-}$ & sextet & 8.7558 & 8.7500 \\
\hline$\left[\mathrm{Fe}^{\mathrm{III}}\left(\mathrm{H}_{2} \mathrm{PO}_{4}\right)_{3}\left(\mathrm{HPO}_{4}\right)\left(\mathrm{H}_{2} \mathrm{O}\right)_{2}\right]^{2-}$ & sextet & 8.7560 & 8.7500 \\
\hline$\left[\mathrm{Fe}^{\mathrm{III}}\left(\mathrm{H}_{2} \mathrm{PO}_{4}\right)_{4}\left(\mathrm{HPO}_{4}\right)\left(\mathrm{H}_{2} \mathrm{O}\right)\right]^{3-}$ & sextet & 8.7556 & 8.7500 \\
\hline
\end{tabular}

Spin contamination for every intermediate was examined. It was found that for most intermediates spin contamination problem is negligible, except for INT1 $\mathbf{1}_{\mathbf{O H} / \mathbf{o H}}$,

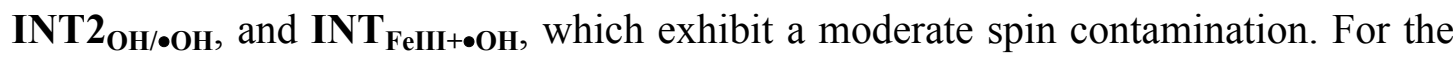
three cases, the ${ }^{\bullet} \mathrm{OH}$ is distant from the $\mathrm{Fe}(\mathrm{III})$ center and, thus, the spin coupling between ${ }^{\circ} \mathrm{OH}$ and five spins on $\mathrm{Fe}(\mathrm{III})$ is very weak. As a consequence, the antiferromagnetic coupling (quintet state) and ferromagnetic coupling (septet state) of these intermediates are very close in energy, resulting in spin-state mixing. ${ }^{53}$ 

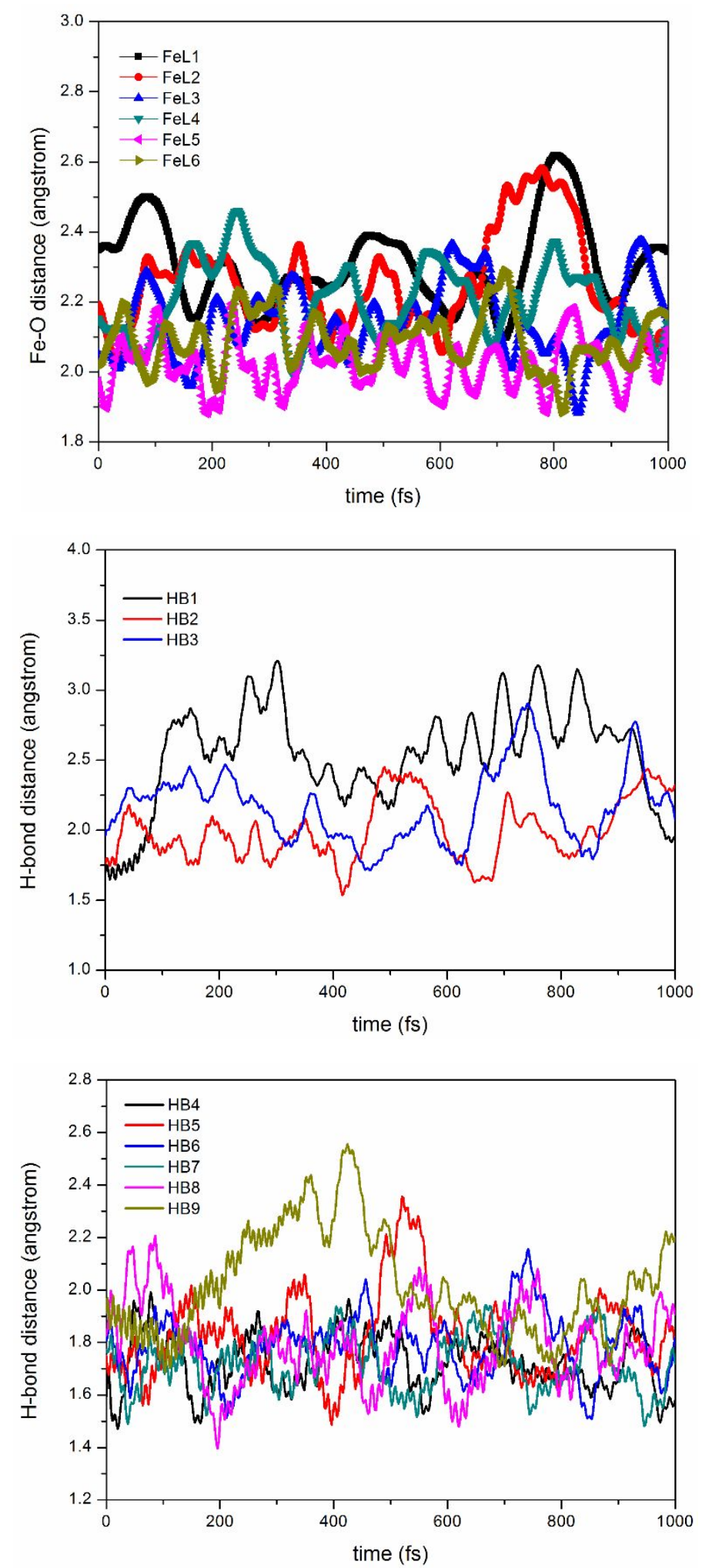

Figure S1. Time evolution of Fe-ligand and H-bond distances for trajectory 2. See Figure 1 for bond label. 

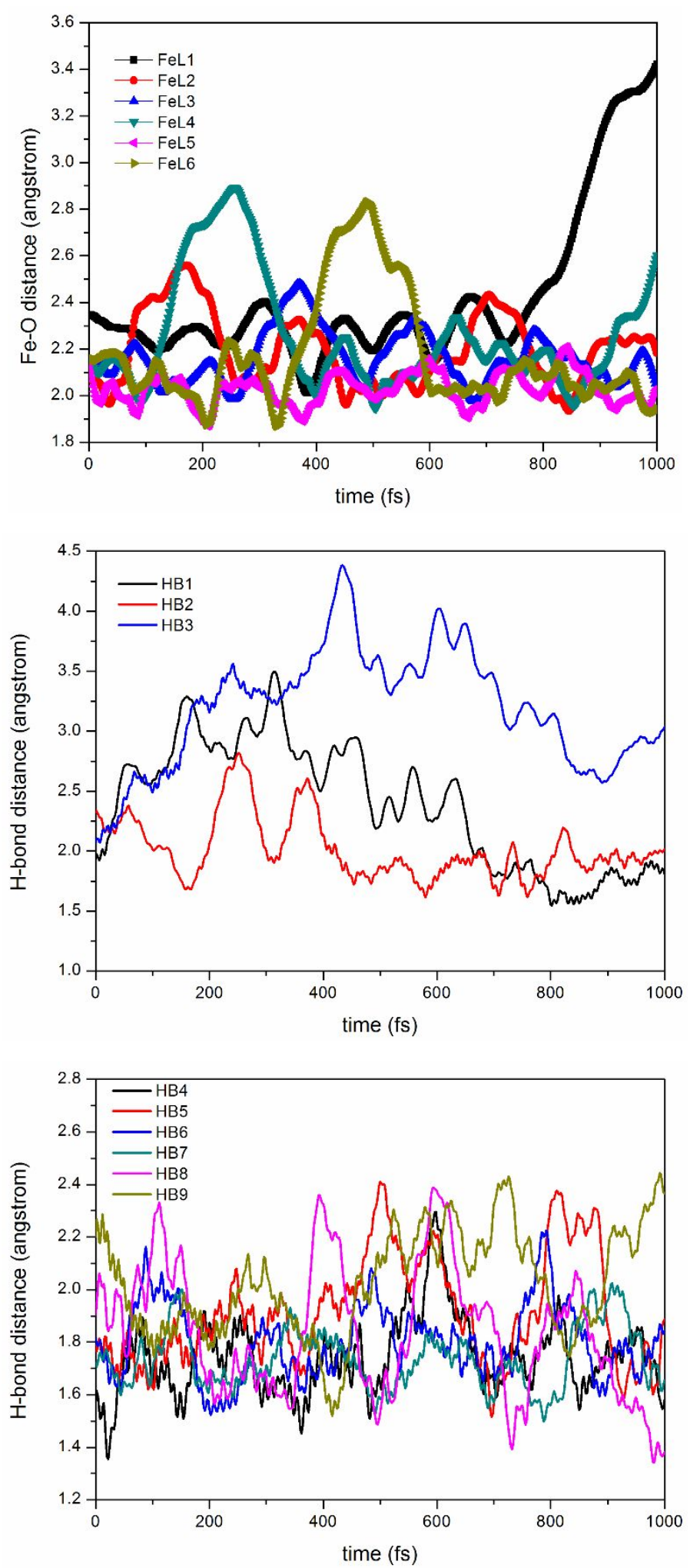

Figure S2. Time evolution of Fe-ligand and H-bond distances for trajectory 3. See Figure 1 for bond label. 